\title{
Diffuse ions coordinate dynamics in a
}

\section{ribonucleoprotein assembly}

\author{
Ailun Wang, ${ }^{\dagger}$ Mariana Levi, ${ }^{\ddagger}$ Udayan Mohanty, ${ }^{*, \dagger}$ and Paul C. Whitford*,+, \\ †Department of Chemistry, Boston College, Chestnut Hill, MA 02467 \\ $\ddagger$ Department of Physics, Northeastern University, Dana Research Center 111, 360 \\ Huntington Ave, Boston, MA 02115 \\ \Center for Theoretical Biological Physics, Northeastern University, 360 Huntington Ave, \\ Boston, MA 02115
}

E-mail: mohanty@bc.edu; p.whitford@neu.edu 


\begin{abstract}
Proper ionic concentrations are required for the functional dynamics of RNA and ribonucleoprotein (RNP) assemblies. While experimental and computational techniques have provided many insights into the properties of chelated ions, less is known about the structural/energetic contributions of diffuse ions. To address this, we present a model that is designed to identify the influence of diffuse ions on the dynamics of biomolecular assemblies. This model employs all-atom (non-H) resolution and explicit ions (monovalent and divalent), where effective potentials account for hydration effects. To benchmark the model, we show that it accurately predicts the number of excess $\mathrm{Mg}^{2+}$ ions for prototypical RNA systems. We then apply it to a bacterial ribosome and find that diffuse ions control the position of the extended L1 stalk region. The simulations also illustrate how diffuse ions facilitate long-range attraction between tRNA and the stalk region. Together, this analysis reveals the direct impact of diffuse ions on the dynamics of an RNP assembly.
\end{abstract}

\title{
Introduction
}

Many large-scale biomolecular processes in the cell depend on the presence of monovalent and multivalent ions. The contribution of cations to structure and dynamics has been documented for a variety of systems, including $\mathrm{RNA}^{1-6} \mathrm{DNA}^{7,8}$ and ribonucleoprotein (RNP) assemblies. ${ }^{9,10}$ A particularly well-characterized RNP assembly is the ribosome, for which specific counterion concentrations are needed for assembly ${ }^{11,12}$ and conformational transitions between functional states. ${ }^{13,14}$ In terms of biological function, in vitro studies have revealed how excess ions can regulate the accuracy of protein synthesis by the ribosome. ${ }^{15,16}$ While the broad influence of ions is acknowledged, identifying the physical-chemical relationship between ions and the mechanical properties of RNP assemblies has remained elusive.

The solvent environment around RNA is generally described as containing chelated and diffuse ions. ${ }^{3,4}$ Chelated (i.e. inner-shell) ions are partially dehydrated, which allows them to 
form strong direct contacts with RNA. ${ }^{3,4,17}$ As a result, chelated ions can remain bound to RNA for ms-s timescales, ${ }^{18-20}$ which exceeds the duration of many biomolecular processes. In contrast, diffuse (outer-shell) ions maintain a coordinated hydration shell (e.g. $\mathrm{Mg}_{2}\left(\mathrm{H}_{2} \mathrm{O}\right)_{6}^{2+}$ ) and associate less strongly with RNA. Even though water molecules can exchange rapidly (microseconds), ${ }^{21-23}$ each ion remains fully hydrated. As a result, the behavior of a diffuse ion is primarily determined by longer-range electrostatic interactions. Despite the transient and weak scale of individual diffuse ion interactions, their collective effect can be significant. For example, tRNA structural stability increases with the concentration of monovalent cations, which can be attributed to screening effects and ion-mediated interactions. ${ }^{24,25}$ The diffuse ionic environment can also control tertiary structure formation in RNA. ${ }^{3}$ A complicating factor when studying diffuse ions is that monovalent and divalent ions competitively associate with RNA. Due to this, a balance of entropic and enthalpic factors can lead to non-trivial relationships between ionic concentrations and biomolecular stability/dynamics.

The essential role of ions in biomolecular systems has motivated their study over a wide range of temporal and spatial scales. There are now many computational approaches available that include implicit-solvent and all-atom explicit-solvent representations. Within the class of implicit-solvent models, there have been applications of non-linear Poisson Boltzmann theory ${ }^{26-28}$ counterions condensation models,${ }^{29-34}$ reference interaction site model ${ }^{35-37}$ and Debye-Hückel (DH) treatments, ${ }^{38-40}$ among other strategies. ${ }^{41-43}$ While these methods can provide accurate representations of the local electric fields, many of them neglect ion-ion correlations, which make it difficult to distinguish between the effects of monovalent and divalent species. ${ }^{44,45}$ In the study of biomolecular assemblies, coarse-grained models frequently employ a DH treatment for monovalent ions. ${ }^{46}$ This is appropriate for describing interactions between opposing charges (e.g. protein-DNA association ${ }^{47,48}$ ), though it can not (by construction) capture ion-induced attraction between polyanionic molecules. ${ }^{49}$ To address this shortcoming, coarse-grained models that include explicit-ion representations have allowed for the analysis of ion-mediated attraction in $\mathrm{DNA}^{50,51}$ and RNA. ${ }^{37,52}$ At a higher level of 
spatial resolution, ion parameters are available for use with all-atom explicit-solvent simulations. ${ }^{53-55}$ These have been used to provided interpretations of ensemble experimental data ${ }^{56}$ and have provided insights into the energetics of small RNA systems. ${ }^{57-59}$ Explicit-solvent techniques can be applied to larger systems, though the associated computational requirements have limited the accessible timescales to microseconds. ${ }^{60}$ Currently, there is not an all-atom model available for which it is tractable to directly connect the properties of diffuse ions with slow (millisecond) conformational processes in large-scale RNP assemblies.

To probe how diffuse ions influence conformational dynamics in biomolecular assemblies, we developed an all-atom (non-Hydrogen atom) model that employs simplified energetics for each biomolecule, along with a transferrable effective potential for explicit monovalent $\left(\mathrm{K}^{+}, \mathrm{Cl}^{-}\right)$and divalent $\left(\mathrm{Mg}^{2+}\right)$ ions. In this model, which we call SMOG-ion, an all-atom structure-based (SMOG) model ${ }^{61,62}$ is used to define intramolecular interactions, while ionic interactions are assigned non-specific effective potentials and Coulomb electrostatics. The ion parameters were first refined based on comparison with explicit-solvent simulations of small model systems (rRNA helix and protein S6). A subset of the ion-RNA parameters were then further refined through comparison with an experimental measure of the excess ionic atmosphere for a prototypical rRNA fragment. While the parameters were defined based on a single experimental concentration, we find the model accurately describes the concentration-dependent properties of the diffuse ionic atmosphere for multiple small RNA molecules. Building upon these benchmarks, we then applied the model to simulate a bacterial ribosome in the presence of monovalent and divalent ions. This analysis reveals how the conformation of the ribosome, specifically the L1 stalk, responds to the diffuse ionic environment. We further demonstrate how diffuse ions mediate recruitment and binding of transfer RNA (tRNA) molecules during the elongation cycle of the ribosome. Together, these calculations provide an initial insight into the relationship between diffuse ions and the structural dynamics of this large biomolecular assembly. 
a
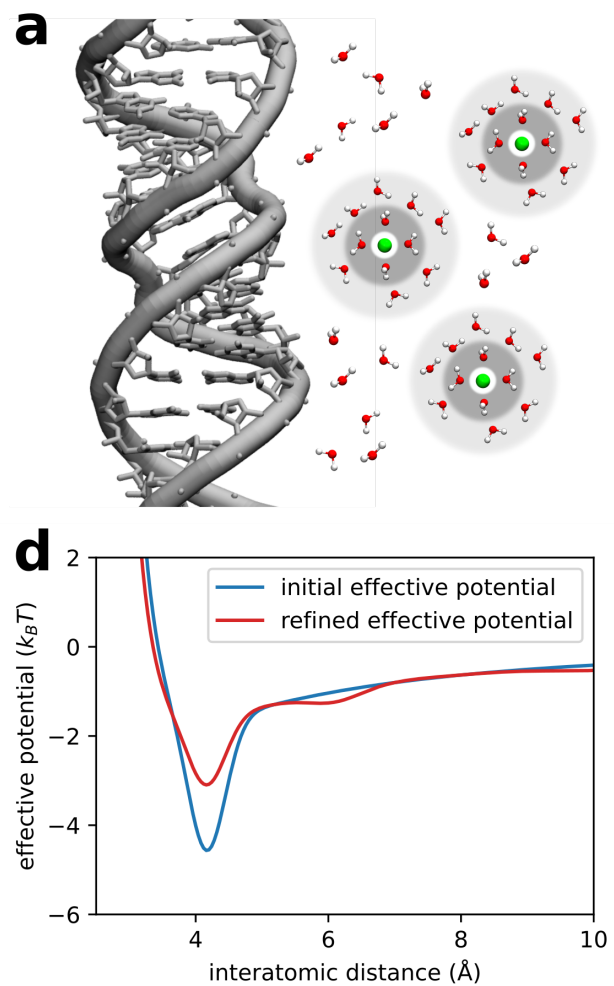

b
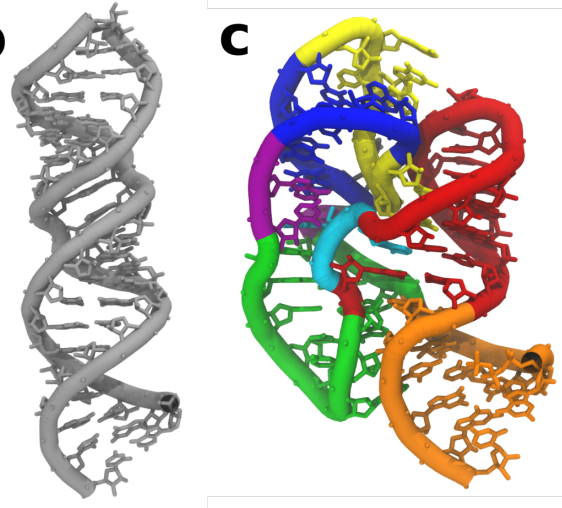

e

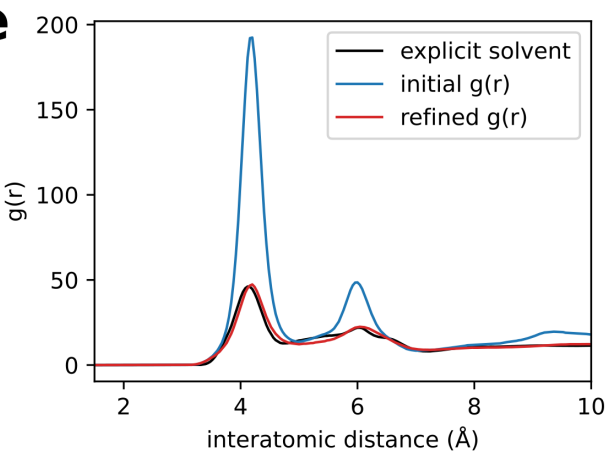

Figure 1: Describing the dynamics of diffuse ions: The SMOG-ion model a) While chelated ions form strong interactions with biomolecules, diffuse ions (green beads) maintain hydration shells (gray rings) that prevent tight binding. Even though diffuse ions associate weakly, due to their large numbers in solution, they can have substantial collective effects on biomolecular energetics. ${ }^{3}$ To study their influence on large molecular assemblies, we developed an all-atom model with simplified energetics (SMOG-ion) in which monovalent and divalent ions are explicitly represented. In this model, intramolecular interactions are defined by a structure-based model, ${ }^{61,62}$ partial charges are assigned to each atom, and effective potentials are introduced to account for the effects of ion-ion correlations and hydration. As benchmarks during parameter refinement, we used explicit-solvent simulations of an rRNA helix (h44; panel b) and protein S6 (Fig. S1), as well and previous experimental measures for the an rRNA 58-mer (panel c). d) Representative interatomic effective potential. Potential for $\mathrm{Mg}^{2+}$ interactions with highly-charge RNA oxygen atoms is shown before (s0 parameters, blue) and after (s1 parameters, red) refinement against explicit-solvent simulations of h44. After refinement, the corresponding radial distribution function (panel e) agrees well with that obtained using the explicit-solvent model, which ensures the ionic shells are consistently described by the models. For comparison of all modeled interactions, see Fig. S3. Minor adjustments to $\mathrm{Mg}^{2+}$ and $\mathrm{K}^{+}$interactions were subsequently introduced based on comparison with experiments for the 58 -mer. ${ }^{63}$ 


\section{Results}

To identify the effects of diffuse ions on the dynamics of biomolecular assemblies, we developed an all-atom model with simplified energetics and explicit ions $\left(\mathrm{K}^{+}, \mathrm{Cl}^{-}, \mathrm{Mg}^{2+}\right)$, which we call SMOG-ion. An all-atom structure-based (SMOG) model ${ }^{61,62}$ with explicit charges defines the biomolecular energetics. Ionic interactions are defined by coulomb electrostatics and effective potentials ( $V_{\mathrm{E}}$, Eq. 3). The "structure-based" terms explicitly favor a pre-defined biomolecular structure, while the effective potentials ensure that the local distribution of ionic species is consistent with in vitro measurements. After describing performance benchmarks and parameterization, we demonstrate how this model may be used to isolate the influence of diffuse ions on biomolecular dynamics. Specifically, we compare the dynamics of the ribosome with multiple variants of the model, which reveals the direct impact of diffuse ions on biomolecular structure and binding.

\section{Simplified model reproduces in vitro ionic distribution}

To parameterize the SMOG-ion model, we first refined the interaction weights based on comparisons with explicit-solvent simulations of multiple systems (Fig. 1 and Fig. S1) for a single ionic composition $\left(\left[\mathrm{MgCl}_{2}\right] \approx 10 \mathrm{mM},[\mathrm{KCl}] \approx 100 \mathrm{mM}\right)$. The explicit-solvent simulations used the Amber99sb-ildn ${ }^{64}$ force field with $\mathrm{Mg}^{2+}$ parameters described by Aqvist ${ }^{53}$ and monovalent ion $\left(\mathrm{K}^{+}\right.$and $\left.\mathrm{Cl}^{-}\right)$parameters of Joung and Cheatham. ${ }^{54}$ We refined the ion-ion, ion-RNA and ion-protein interactions in our model using the procedure of Savelyev and Papoian, ${ }^{50}$ where linear parameters in the Hamiltonian are iteratively updated through use of a first-order expansion of the partition function (Eq. 2b in SI). With regards to nomenclature, "s $N$ " denotes the model parameters after refinement step $\underline{N}$. The initial parameters (s0; Fig. 1d, blue curve) were estimated based on inspection of radial distribution functions (RDFs) calculated from explicit-solvent simulations (Fig. S2a). With the s0 parameters, the positions and widths of the peaks in each RDF were consistent with the explicit-solvent model 
(Fig. 1e, blue curve), though there were significant differences in the relative heights of each peak. We then sequentially refined the ion-ion, ion-RNA and ion-protein interactions (i.e. s1 parameter set; details in Supporting Information). The s1 model produced RDFs for $\mathrm{Mg}^{2+}$, $\mathrm{K}^{+}$and $\mathrm{Cl}^{-}$that exhibited excellent agreement with those obtained with the explicit-solvent model (Figs. 1e and S3). This initial refinement ensures the description of outer ionic shells is comparable to that predicted by a more highly-detailed model.

In the second stage of refinement, a subset of the ion parameters was adjusted based on comparison with experimental measures of diffuse ionic distributions. For this, the preferential interaction coefficient of $\mathrm{Mg}^{2+}$ ions $\left(\Gamma_{2+}\right)$ was calculated from simulations of a 58nucleotide rRNA fragment ${ }^{65}$ (Fig. 1c and Fig. S4a). For consistency with the experimental conditions, ${ }^{63}$ we simulated the 58 -mer with $\left[\mathrm{MgCl}_{2}\right]=1 \mathrm{mM}$ and $[\mathrm{KCl}]=150 \mathrm{mM}$. Even though the s1 model parameters recapitulate the ionic distributions predicted by the explicitsolvent model (Fig. 1e), $\Gamma_{2+}$ was significantly underestimated for the 58-mer. Specifically, the predicted value of $\Gamma_{2+}$ was $2.2 \pm 0.6$, whereas the experimental value was $10.4 .^{63}$

Since underestimation of $\Gamma_{2+}$ indicated an imbalance between $\mathrm{K}^{+}$and $\mathrm{Mg}^{2+}$ association strengths with RNA, ${ }^{3}$ we introduced minor changes to the $\mathrm{Mg}^{2+}$ and $\mathrm{K}^{+}$interactions with highly-electronegative RNA atoms. In explicit-solvent simulations of RNA, excess $\mathrm{K}^{+}$ions are frequently partially dehydrated, which can artificially amplify the effective strength of $\mathrm{K}^{+}$-RNA interactions. ${ }^{66,67}$ This observation is at odds with NMR studies that have reported most $\mathrm{K}^{+}$ions tend to remain fully hydrated. ${ }^{17}$ Since the s1 parameter set was based on comparison with an explicit-solvent model, we removed the effective potential that stabilizes short-range (first outer shell) interactions between diffuse $\mathrm{K}^{+}$ions and electronegative $(q<-0.5) \mathrm{O}$ and $\mathrm{N}$ atoms (yielding the s2 parameter set). While this increased $\Gamma_{2+}$ from $2.2(\mathrm{~s} 1)$ to $6.4(\mathrm{~s} 2)$, the persistent underestimation of $\Gamma_{2+}$ suggested the effective potential for $\mathrm{Mg}^{2+}$ was also insufficiently stabilizing. Since $\Gamma_{2+}$ is predominantly influenced by the distribution of diffuse $\mathrm{Mg}^{2+}$ ions around highly electronegative atoms, ${ }^{37,66}$ we introduced a small increase to the stability of these interactions in our model. Based on preliminary free-energy 
perturbation analysis (not shown), we increased the strength of the short-range (outer shell) $\mathrm{Mg}^{2+}$ interaction by $0.16 \epsilon\left(\mathrm{s} 3\right.$ parameter set) ${ }^{\mathrm{i}}$. These parameters predicted $\Gamma_{2+}=9.5 \pm 0.7$, which is comparable to the experimental value of 10.4. When considering the uncertainty in the calculated $\Gamma_{2+}$ values (see Methods), we decided to terminate the refinement process upon reaching this level of agreement. For all subsequent analysis, we used the s3 parameter set, which will simply be referred to as the SMOG-ion model.

\section{Spatial partitioning of diffuse ionic species}

Our simplified model predicts condensation and spatial partitioning of ionic species, which is consistent with monovalent and divalent ions contributing differentially to the stability of secondary and tertiary structure in RNA. The predicted spatial distributions (Fig. 2) for the 58-mer are generally corroborated by prior crystallographic analysis and explicitsolvent simulations. In terms of biomolecular structure, $\mathrm{K}^{+}$ions primarily populate the major grooves of the RNA, while $\mathrm{Mg}^{2+}$ ions appear to bridge interactions associated with tertiary structure.

Before comparing the predicted ionic distributions with crystallographic data, it is necessary to describe the experimental assignment of ions. The crystal structure of the 58mer $^{65}$ (PDB ID: 1HC8) contains two asymmetric protein-RNA assemblies in the unit cell, and there are eight $\mathrm{Mg}^{2+}$ assignments that are common (Fig. 2d, pink beads) ${ }^{\mathrm{ii}}$. Of these common assignments, Poisson-Boltzmann calculations indicate one is likely to be bound (Fig. 2d, circled. binding free energy $\Delta G_{\text {bind }}=-4.8 \mathrm{kcal} / \mathrm{mol}^{68}$ ). Based on this, the system is typically described as possessing a single chelated $\mathrm{Mg}^{2+}$ ion. ${ }^{65}$ Since our model was parameterized for the study of diffuse ions, harmonic interactions were introduced to maintain the position of this single chelated $\mathrm{Mg}^{2+}$ ion. There is also a chelated $\mathrm{K}^{+}$(calculated

\footnotetext{
${ }^{\mathrm{i}}$ The reduced energy unit $\epsilon$ is equal to $2 k_{\mathrm{B}} T$. For $\mathrm{Mg}^{2+}-\mathrm{O}$ interactions, $B_{1}$ was changed from -0.73 to -0.89 . For $\mathrm{Mg}^{2+}-\mathrm{N}$ interactions, it was changed from -0.86 to -1.02 .

${ }^{\text {ii }} 21$ coordinates of $\mathrm{Mg}^{2+}$ ions were reported in the two asymmetric assemblies of the 58-mer (chain $\mathrm{C}$ and chain D in PDB 1HC8). Alignment of the monomers reveals 13 distinct binding positions, where 8 are occupied in both RNA molecules.
} 

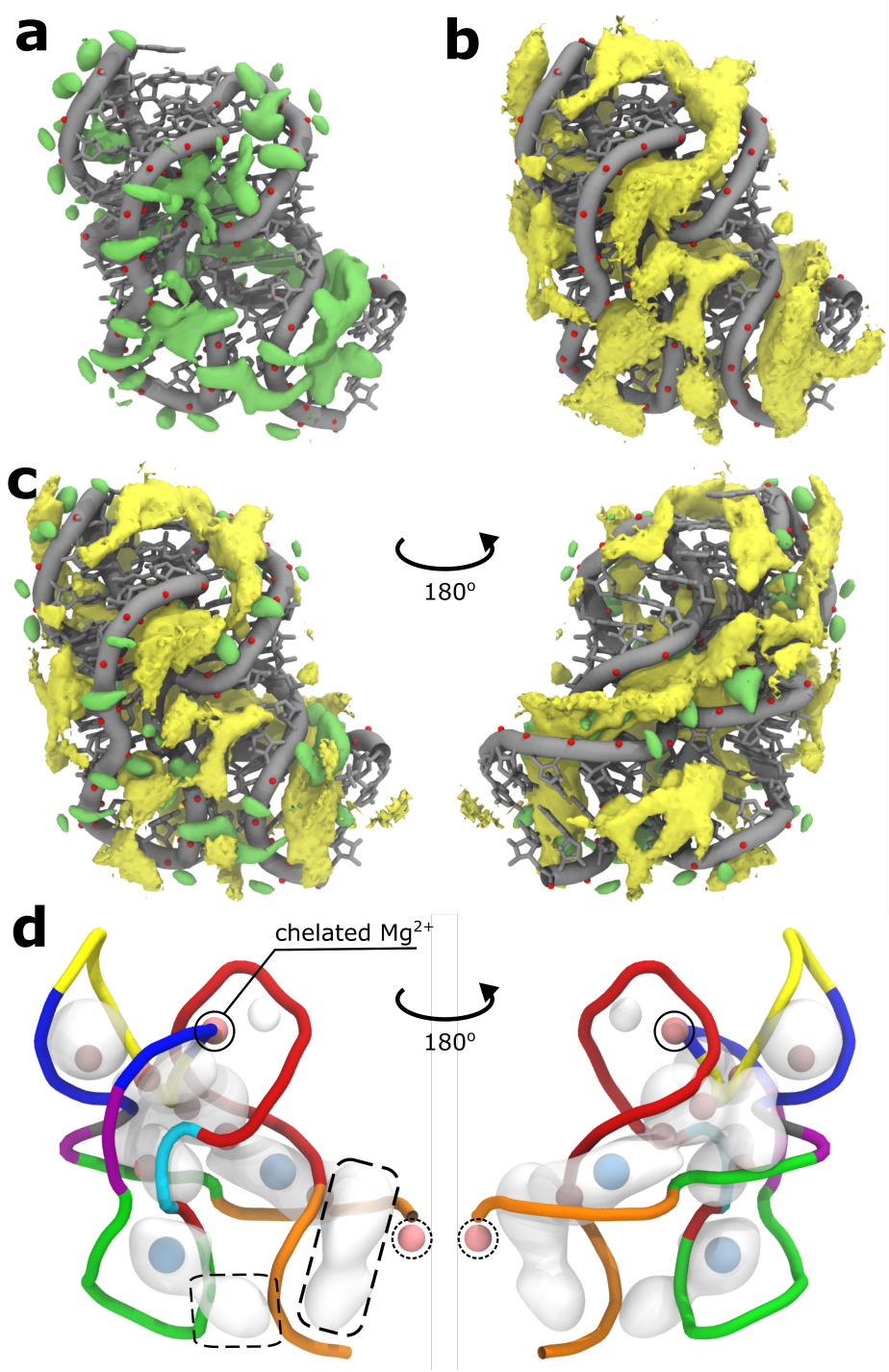

Figure 2: Characteristics of monovalent and divalent ion-RNA association a) Spatial distribution function (SDF) of diffuse $\mathrm{Mg}^{2+}$ ions for the $58-\operatorname{mer}\left(\left[\mathrm{MgCl}_{2}\right]=1 \mathrm{mM}\right.$, $[\mathrm{KCl}]=150 \mathrm{mM})$. The isosurface represents a $1.3 \mathrm{M}$ concentration of $\mathrm{Mg}^{2+}(1300$-fold enrichment over bulk). b) SDF for diffuse $\mathrm{K}^{+}$ions (isosurface at 1.3M). c) The difference between the SDFs : $\Delta \rho=\rho_{\mathrm{Mg}^{2+}}-\rho_{\mathrm{K}^{+}}$, where $\rho_{\mathrm{i}}$ is the SDF of ion type i. The green isosurface shows preferential association of $\mathrm{Mg}^{2+}$ ions $(\Delta \rho=1.3 \mathrm{M})$, while yellow shows a preference for $\mathrm{K}^{+}$ ions $(\Delta \rho=-1.3 \mathrm{M}) . \mathrm{K}^{+}$tends to populate the RNA grooves, consistent with its significant influence on the stability of secondary structure. ${ }^{1} \mathrm{Mg}^{2+}$ ions are dominant along the RNA backbone, consistent with their contribution to tertiary structure formation. ${ }^{2,3}$ d) SMOG-ion model predicts population of crystallographically-reported ionic densities. SDF calculated for diffuse $\mathrm{Mg}^{2+}$ ions (chelated ion not included) after applying a Gaussian filter. Isosurface shown for $500 \mathrm{mM}$. Crystallographically assigned non-chelated $\mathrm{Mg}^{2+}$ ions (pink) and $\mathrm{Os}^{3+}$ ions (blue) are within the predicted regions of high diffuse $\mathrm{Mg}^{2+}$ densities, except a solitary $\mathrm{Mg}^{2+}$ ion near the terminal tail (dashed circle). In experiments, crystallographic contacts with the tail likely facilitate ion localization. In addition to predicting the crystallographic ions, there are two additional regions of high density (dashed boxes), which may further contribute to tertiary structure formation. 
$\Delta G_{\text {bind }}=-30 \mathrm{kcal} / \mathrm{mol}^{65}$ ) that is restrained to its binding site. Finally, the crystal structure has two non-chelated $\mathrm{Os}^{3+}$ ions that were not included in our simulations.

The SMOG-ion model predicts high local concentrations of $\mathrm{Mg}^{2+}$ ions that coincide with the crystallographically-resolved positions of non-chelated multivalent ions. Specifically, the model predicts regions of high $\mathrm{Mg}^{2+}$ density that overlap with six of the seven non-chelated $\mathrm{Mg}^{2+}$ positions (Fig. 2). The only outlier is positioned near the terminal guanosine-5'triphosphate residue (Fig. 2d, dashed circle). In the simulations, mobility of the tail impedes ion association. In contrast, in the experimental structure, crystallographic contacts with the tail likely reduce the flexibility and facilitate a higher ion density. The model also predicts high $\mathrm{Mg}^{2+}$ densities that overlap with the experimentally assigned positions of both $\mathrm{Os}^{3+}$ ions (Fig. 2d, blue beads). Overall, consistency between the predicted SDFs and crystallographic analysis suggests that our model provides an accurate description of the local ionic environment.

The SMOG-ion model implicates differential contributions of monovalent and divalent ions to the stability of RNA. In our model, diffuse $\mathrm{K}^{+}$and $\mathrm{Mg}^{2+}$ ions both populate the major groove of double-stranded RNA helices (Fig. 2a,b), as predicted by explicit-solvent simulations. ${ }^{69,70}$ This shows how both ionic species can contribute to the stability of secondary structure in RNA. Consistent with explicit-solvent simulations, ${ }^{66}$ we also find that $\mathrm{Mg}^{2+}$ ions accumulate around each backbone phosphate group (Fig. 2c, green). Thus, as expected, $\mathrm{Mg}^{2+}$ ions appear to be a primary contributor to tertiary structure formation in RNA. Interestingly, the model predicts two regions of high $\mathrm{Mg}^{2+}$ density that do not coincide with assigned ion positions (Fig. 2d, boxed regions). Both of these regions span RNA segments that are distant in sequence. These high-density regions further suggest how $\mathrm{Mg}^{2+}$ ions may facilitate higher-order structure formation in RNA. 


\section{Model captures concentration-dependent ionic atmosphere}

Before applying the SMOG-ion model to a large biomolecular assembly, we evaluated its transferability by comparing the predicted concentration dependence of $\Gamma_{2+}$ with experimental values for two well-studied RNA systems: the 58-mer rRNA fragment ${ }^{63}$ and an adenine riboswitch. ${ }^{71}$ Comparing concentration-dependent values of $\Gamma_{2+}$ allows one to ask whether the modeled parameters accurately describe the competition of ionic species. It is important to note that the ion-RNA interaction strengths were assigned based on comparison with explicit-solvent simulation and a single experimental value of $\Gamma_{2+}$. Accordingly, calculating $\Gamma_{2+}$ values for multiple systems over a range of ion concentrations represents a test of the transferability of the model.

We first compared predicted and experimental ${ }^{63}$ values of $\Gamma_{2+}$ for the 58 -mer rRNA (Fig. 3a,b). While the model was calibrated using the 58-mer with $\left[\mathrm{MgCl}_{2}\right]=1 \mathrm{mM}$ and $[\mathrm{KCl}]=150 \mathrm{mM}$, we find it accurately predicts the change in $\Gamma_{2+}$ as a function of $\left[\mathrm{MgCl}_{2}\right]$. Over the studied concentrations $(\sim 0.2-1 \mathrm{mM})$, the experimental $\Gamma_{2+}$ values change by 6.5 , whereas the model predicts a change of 5.5. There is a systematic underestimation $(\sim 1)$ of the $\Gamma_{2+}$ values at higher ion concentrations, though the statistical uncertainty in the theoretical values is comparable to this difference. Here, we compare our results to the experimental values obtained with a hyperstable variant of the rRNA (U1061A), and we limit our comparisons to higher experimental ionic concentrations. Over this range the RNA is known to remain folded, which allows our comparisons to specifically assess the accuracy of the ion parameters, independent of the biomolecular potential. Nonetheless, there is a possibility that the experimental values also reflect concentration-dependent effects on RNA structure that are not addressed here. With these experimental and statistical uncertainties in mind, one may consider the residual differences between $\Gamma_{2+}$ values to be minor.

Applying the model to the adenine riboswitch ${ }^{\text {iii }}$ (Fig. 3c) demonstrates the transferability of the ion parameters. Since the riboswitch was not utilized for any aspect of parameter

\footnotetext{
${ }^{\text {iii }}$ with ligand bound
} 


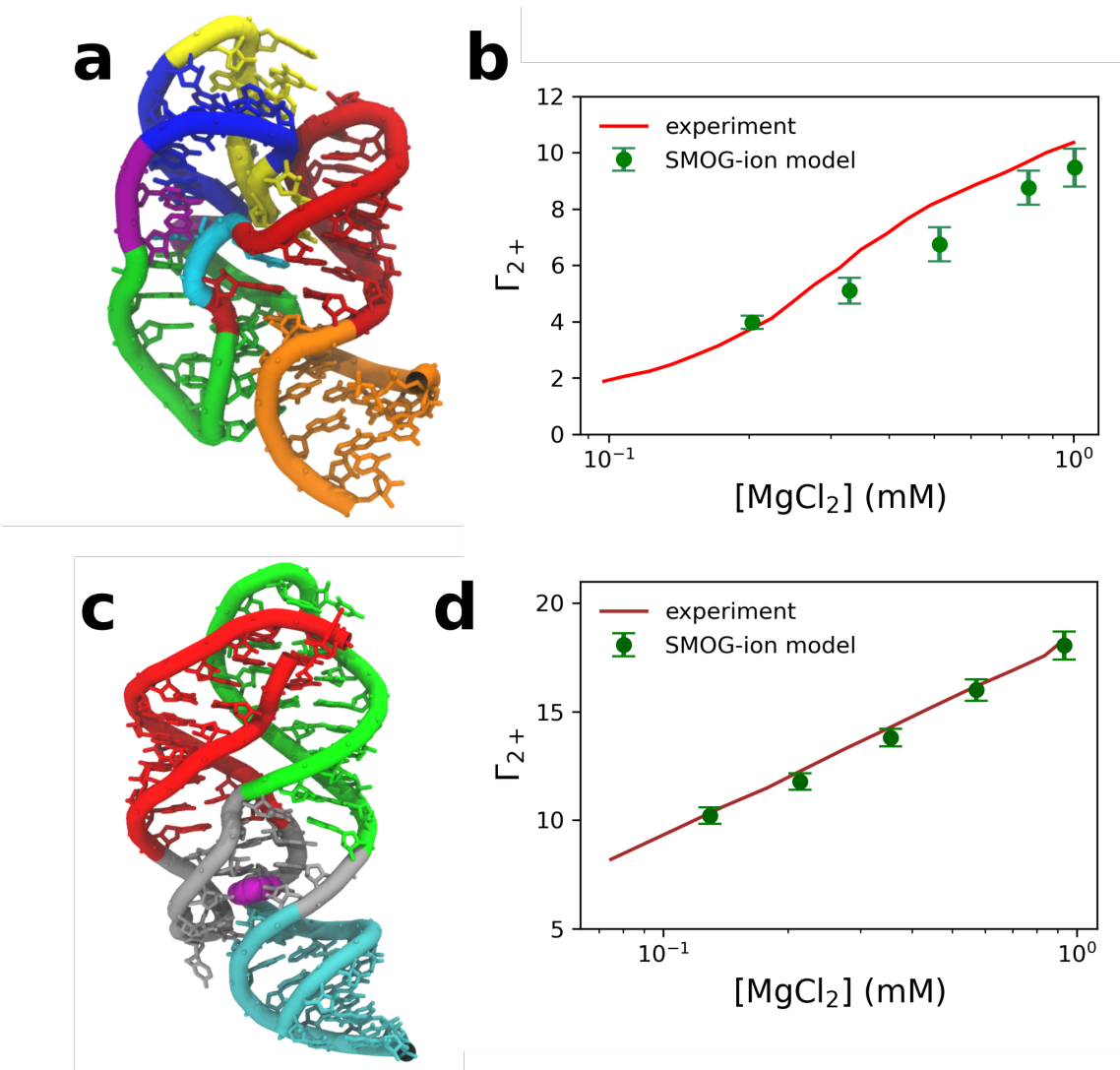

Figure 3: Simplified model captures concentration-dependent ionic distributions a) Tertiary structure of the 58-mer rRNA fragment ${ }^{65}$ (colored as in Fig. 1c). b) Comparison of experimental ${ }^{63}$ and simulated values of the preferential interaction coefficient $\left(\Gamma_{2+}\right)$ for the 58 -mer $([\mathrm{KCl}]=150 \mathrm{mM})$. While the model was parameterized based on comparison with the experimental value of $\Gamma_{2+}$ at $\left[\mathrm{MgCl}_{2}\right]=1 \mathrm{mM}$, the predicted concentration dependence follows the experimental behavior. c) Tertiary structure of the adenine riboswitch, ${ }^{71}$ colored as in Fig.S4b. d) Experimental ${ }^{71}$ and predicted values of $\Gamma_{2+}$ for the adenine riboswitch $([\mathrm{KCl}]=50 \mathrm{mM})$ exhibit excellent agreement. This represents a blind test of the model, since the riboswitch was not used during model parameterization. Further, the SMOG-ion model parameters were established using benchmark systems that were at higher value of [KCl] (100-150 vs. $50 \mathrm{mM})$. Accordingly, these comparisons illustrate the transferrability of the model to other RNA systems and ionic concentrations. Error bars in (b) and (d) represent the standard deviation of $\Gamma_{2+}$ calculated from 20 replicate simulations. 
refinement, it serves as a blind test of the predictive capabilities of the model. In addition, the experiments were performed at lower $[\mathrm{KCl}]$ values for the adenine riboswitch than for the 58 -mer $\left(50 \mathrm{mM}^{71}\right.$ vs. $150 \mathrm{mM}^{63}$ ). Therefore, this comparison implicitly evaluates the predicted concentration-dependent influence of monovalent ions on $\mathrm{Mg}^{2+}-\mathrm{RNA}$ association. We find that the predicted $\Gamma_{2+}$ values agree very well with experimental measurements, where the differences are less than 0.3 (Fig. 3d). Combined with our analysis of the 58-mer, this demonstrates the ability of the SMOG-ion model to accurately estimate the energetics and distribution of diffuse ions.

\section{Diffuse ions control ribosome conformation}

Simulations with the SMOG-ion model reveal how diffuse ions can influence the conformational properties of a well-studied RNP assembly: the ribosome. The importance of chelated ions to ribosome structure is well known, ${ }^{11}$ and recent structural studies have been able to identify the precise composition of the bound ions. ${ }^{72}$ To complement insights into bound ions, simulations performed with the SMOG-ion model illustrate how diffuse ions contribute to the dynamics of large structural elements within the ribosome. Specifically, we find that diffuse ions help position the L1 stalk (Fig. 4) and facilitate recruitment of tRNA molecules by the stalk.

To dissect the energetic factors that control the ribosomal L1 stalk, we performed simulations of the full $70 \mathrm{~S}$ ribosome using multiple variants of the SMOG model. In order to identify the direct influence of diffuse ions on the position of the L1 stalk, this initial analysis did not consider an E-site tRNA molecule (Fig. 4a). As expected, when a conventional (electrostatics-free) all-atom structure-based model (SMOG model; Tab. 1) ${ }^{61,62}$ was applied, the average position of the stalk aligned with the crystallographic structure (Fig. S5a). We next introduced Coulomb electrostatic interactions in the absence of diffuse ions (SMOGcoulomb model; Tab. 1). Here, repulsion between negatively charged rRNA residues leads to the adoption of an exaggerated outward/extended configuration of the stalk (Fig. 4b), 


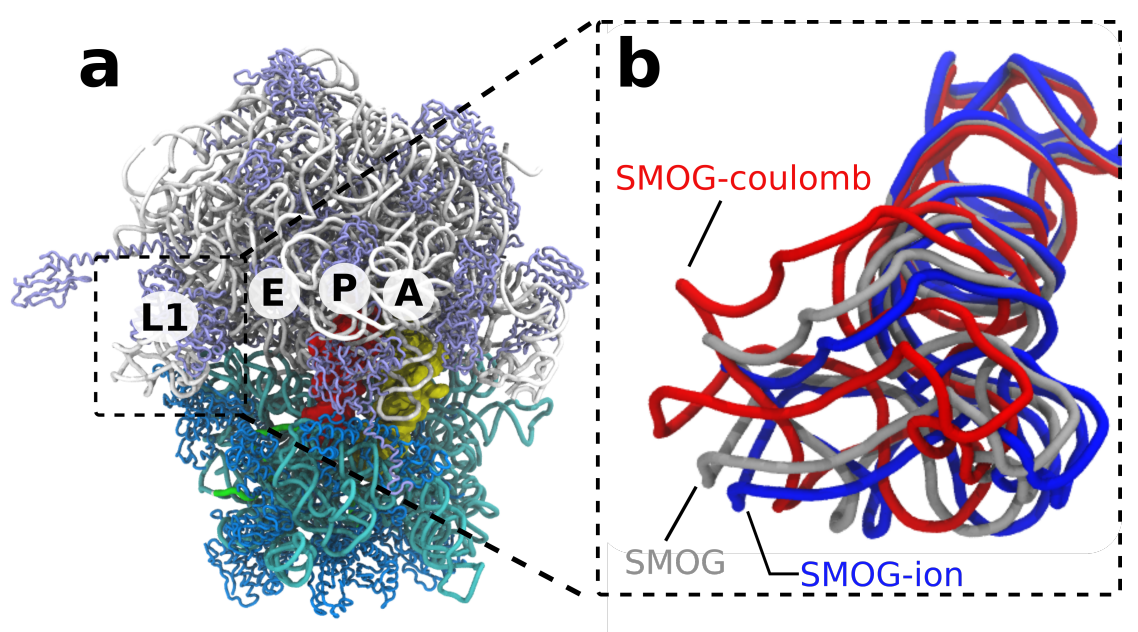

Figure 4: Diffuse ions induce conformational rearrangements in the ribosome a) Crystallographic structure of the bacterial ribosome (PDB: 6QNR ${ }^{72}$ ). During elongation, tRNA molecules (red, yellow) sequentially bind the A, P and E sites. During tRNA translocation (P-to-E site displacements), the L1 stalk (boxed) can bind and facilitate tRNA movement. b) Average structures, calculated from simulations with multiple variants of the all-atom structure-based model. When an electrostatics-free SMOG model is used, the L1 stalks maintains a position (gray) that is consistent with the crystallographic structure (Fig. S5a). When electrostatic interactions are included, in the absence of diffuse ions, the L1 stalk adopts a more extended/outward configuration (red). This may be attributed to chargeinduced backbone repulsion that disfavors the B-form RNA structure. In contrast, when diffuse ions are included with the SMOG-ion model, the stalk adopts a more inward conformation, relative to the electrostatics-free case. This reveals the strong effect that diffuse ions can have on the dynamics of extended structural elements in the ribosome. 
where the average position ${ }^{\text {iv }}$ is displaced by $\sim 14 \AA$, relative to the crystallographic structure. This extended orientation was also observed when screening effects of monovalent ions were taken into account through use of a Debye-Hückel (DH) treatment (Fig. S5b, pink). Since DH theory only considers screening effects, and not ion-mediated interactions, this extended position of L1 is expected.

Before discussing the influence of explicit diffuse ions, it is important to explain why the stalk remains nominally folded when using a diffuse-ion-free representation. First, while diffuse ions are absent, bound ions are included and harmonically restrained. Accordingly, a significant degree of repulsive RNA-RNA interactions are attenuated by the bound ions. Thus, these diffuse-ion-free representations are not intended to mimic ion-free experimental conditions. Instead, they serve as points of comparison for simulations that include diffuse ions (below). The second contributor to this feature is that the commonly-used SMOG model overestimates biomolecular stability. ${ }^{73}$ As a consequence, the diffuse-ion-free models lead to deformations of L1, rather than full unfolding. Accordingly, while the SMOG-ion model can accurately describe structural fluctuations under non-zero ion concentrations, further refinement will be needed to extend the applicability of the model to investigate RNA stability and association of chelated ions.

In stark contrast with the ion-free (SMOG-coulomb) simulations, when the diffuse ionic environment is explicitly described (SMOG-ion model), the L1 stalk favors positions that are inward (Fig. 4b, blue). The only difference between the SMOG-coulomb and SMOG-ion models is that diffuse ions are included in the latter. Accordingly, comparing the dynamics with these models reveals how diffuse ions directly coordinate this large-scale structural feature of the ribosome. With regard to interpretation, the precise position of the stalk will be due to ion-mediated interactions, as well as the underlying structure-based model. Since the structure-based model can be defined based on any ribosome structure, the final position of the stalk should not be considered a firm prediction. However, comparing the dynamics with

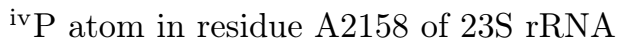


diffuse-ion-absent and diffuse-ion-present models unambiguously demonstrates the major impact diffuse ions can have on biomolecular structure.

Table 1: Models used to simulate the ribosome

\begin{tabular}{lcc}
\hline model name & charge interactions & num. diffuse ions \\
\hline SMOG & no charges & 0 \\
SMOG-coulomb & Coulomb & 0 \\
SMOG-DH & Debye-Hückel & 0 \\
SMOG-ion & Coulomb + eff. pot. & 47800 \\
\hline
\end{tabular}

Since diffuse ions can influence the position of the L1 stalk, they are likely to have a profound impact on the biological dynamics of the assembly. During protein synthesis, tRNA molecules rapidly $\left(\sim 10-100\right.$ milliseconds $\left.{ }^{74,75}\right)$ traverse three distinct ribosomal binding sites (A, $\mathrm{P}$ and $\mathrm{E}$ ). These rearrangements are facilitated by interactions with the L1 stalk, which transiently binds tRNA during translocation and exit. ${ }^{76-81}$ The dynamic character of the L1 stalk has been highlighted by elastic network models ${ }^{82,83}$ and explicit-solvent simulations, ${ }^{84}$ which predict large-scale structural fluctuations are energetically accessible. This high degree of flexibility is consistent with the body of structural ${ }^{76-78,85}$ and $\operatorname{smFRET}^{79-81}$ data that implicates a balance between "open" (outward) and "closed" (inward) conformations of the L1 stalk during tRNA translocation. The current calculations suggest diffuse ions have a central role in determining the balance between more inward and outward conformations of the stalk. In doing so, diffuse ions are likely to impact the ability of tRNA molecules to undergo translocation and/or exit the ribosome.

\section{Diffuse divalent ions mediate tRNA recruitment}

In addition to coordinating the structure of the ribosome, we find that diffuse ions can mediate recruitment of tRNA molecules by the L1 stalk. Here, we repeated all simulations with the E-site tRNA molecule included. To isolate the influence of diffuse ions, we compared the structural and energetic properties of the ribosome when simulations were performed with 
multiple variants of the model (electrostatics-free, ion-free electrostatics, screened electrostatics and explicit ions; Tab. 1 and Tab. 2).

Simulations with the E-site tRNA further highlight the strong influence of diffuse ions on the position of the L1 stalk. When the ribosome is simulated with the electrostatic-free SMOG model, ${ }^{61,62}$ the stalk is shifted slightly away from the E-site tRNA (Tab. 2, Fig. S6b). While the crystallographic structure is defined to be stable, attractive interactions between L1 and tRNA were not included in this implementation of the model. Due to this, the bulky character of L1 leads to an effective repulsion that arises from excluded volume interactions. This effect is amplified when coulomb electrostatics are included without diffuse ions (SMOGcoulomb model; Fig. S6b). In that case, the L1 stalk is displaced an additional $\sim 9 \AA$ from the tRNA, while a smaller displacement was observed when screened electrostatics was included (SMOG-DH model; Fig. S6b). For both electrostatic representations, displacement of the stalk may be partially attributed to repulsion between backbone atoms of the L1 stalk and tRNA. In contrast, when explicit diffuse ions are included (SMOG-ion model; Fig. S6b), the L1 stalk remains near the tRNA molecule.

The SMOG-ion model indicates that energetic stabilization between tRNA and L1 is directly imparted by ion-mediated interactions. To quantify this effect, we calculated the potential energies associated with interactions between L1 and tRNA $\left(U_{\mathrm{L} 1-\mathrm{tRNA}}\right)$, L1 and the diffuse ions $\left(U_{\mathrm{L} 1-\text { ions }}\right)$, as well as between the E-site tRNA and the diffuse ions $\left(U_{\text {tRNA-ions }}\right)$. To compare these energies, we considered the average of each as a function of the distance between the L1 stalk and E-site tRNA: $R_{\text {L1-tRNA }}$ (distance between P atoms of A2112 in the rRNA and G19 in the tRNA; Fig. S7). As the tRNA approaches the L1 stalk $\left(R_{\text {L1-tRNA }}\right.$ decreasing from $\sim 20 \AA$ to $\sim 10 \AA$ ), $U_{\mathrm{L} 1-\mathrm{tRNA}}$ monotonically increases by $\sim 10 \mathrm{k}_{\mathrm{B}} \mathrm{T}$ (Fig. $5 \mathrm{c}$ ). This is consistent with direct repulsion arising from the negatively charged RNA backbones. However, the ion-associated energies $U_{\mathrm{L} 1-\text { ions }}$ and $U_{\text {tRNA-ions }}$ decrease by $\sim 20 \mathrm{k}_{\mathrm{B}} \mathrm{T}$ and $\sim 10$ $\mathrm{k}_{\mathrm{B}} \mathrm{T}$, respectively. Accordingly, the diffuse ions do not simply neutralize the repulsive character of L1-tRNA interactions. Instead, there is a net attraction between the stalk and 
Table 2: Summary of displacement of L1 stalk with the presence of E-site tRNA in ribosome using varied simplified models.

\begin{tabular}{|c|c|c|c|}
\hline \multirow[t]{2}{*}{ model } & \multirow{2}{*}{$\begin{array}{c}\text { E-site tRNA absent } \\
\text { displacement of } \\
\text { L1 stalk }(\AA)^{\mathrm{a}}\end{array}$} & \multicolumn{2}{|c|}{ E-site tRNA present } \\
\hline & & $\begin{array}{c}\text { displacement } \\
\text { of L1 } \\
\operatorname{stalk}(\AA)^{\mathrm{a}}\end{array}$ & $R_{\text {L1-tRNA }}(\AA)^{\mathrm{b}}$ \\
\hline crystal structure & 0 & 0 & 10.3 \\
\hline SMOG & 6.7 & 10.1 & 14.6 \\
\hline SMOG-coulomb & 13.9 & 19.7 & 19.4 \\
\hline SMOG-DH & 10.5 & 16.5 & 17.8 \\
\hline SMOG-ion & $-8.3^{\mathrm{c}}$ & 4.2 & 12.3 \\
\hline \multicolumn{4}{|c|}{$\begin{array}{l}\text { a Measured by the atomic distance of atom P in residue A2158 of } 23 \mathrm{~S} \text { rRNA from its } \\
\text { position in crystal structure. } \\
\text { b Measured by the distance between atom P in residue G19 of E site tRNA and atom } \\
\mathrm{P} \text { in residue G2112 in } 23 \mathrm{~S} \text { rRNA (Fig. S7). Probability distributions shown in Fig. S8. } \\
\text { c Negative number means L1 stalk has inward displacement in comparison with its } \\
\text { crystallographic position. }\end{array}$} \\
\hline
\end{tabular}

E-site tRNA that is introduced by the diffuse ionic environment. These calculations suggest that diffuse ions mediate long-range attraction and recruitment of tRNA by the stalk. This initial ion-guided approach can position the tRNA and stalk, such that precise short-range interactions may be formed (base stacking, van der Waals, etc). ${ }^{84,86}$

To conclude our analysis, we asked whether ion-mediated effects are primarily due to monovalent or divalent ions. To address this, we calculated the spatial distribution functions of $\mathrm{Mg}^{2+}$ and $\mathrm{K}^{+}$ions around the L1 stalk (Fig. 5 and Fig. S9). We find that the $\mathrm{Mg}^{2+}$ ions exhibit dispersed spatial distributions that span the major groove of the rRNA and tRNA. While high-density regions are visible for $\mathrm{Mg}^{2+}$, the density of $\mathrm{K}^{+}$ions is much lower (Fig. S9). These spatial depictions may be quantified in terms of the number of $\mathrm{Mg}^{2+}$ and $\mathrm{K}^{+}$that

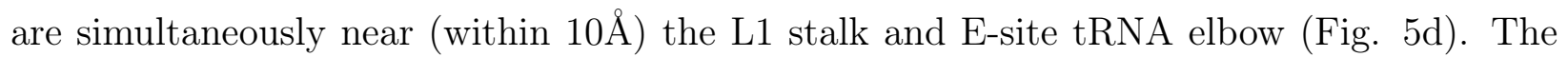
mean number of $\mathrm{Mg}^{2+}$ that bridge L1 and tRNA is 11.6, while the mean number of $\mathrm{K}^{+}$ions is only 2.4 (Fig. 5d). This indicates that $\mathrm{Mg}^{2+}$ ions accumulate near the L1-tRNA interface and expel $\mathrm{K}^{+}$ions. As a result, recruitment of tRNA by L1 appears to be controlled by 

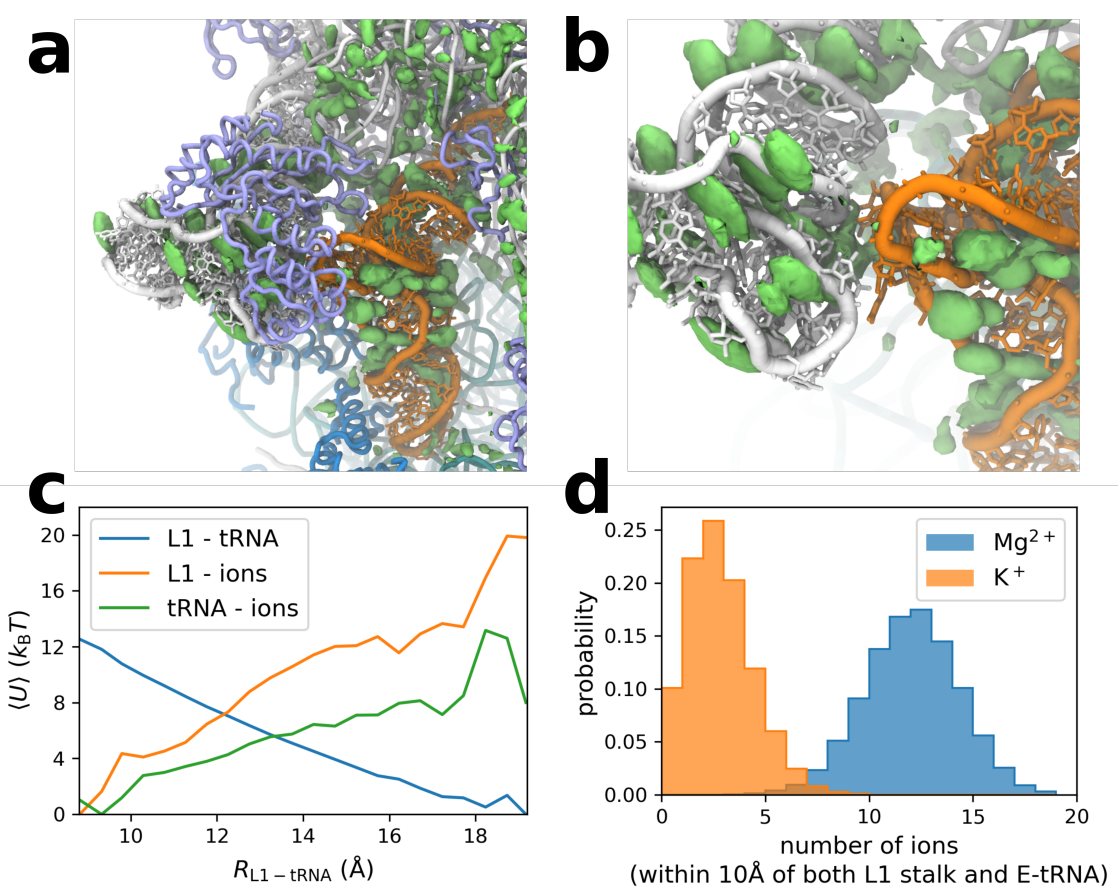

Figure 5: $\mathbf{M g}^{2+}$ ions mediate ribosome-tRNA association a) SDF of $\mathrm{Mg}^{2+}$ ions (green surface; conc. 3M) in the vicinity of L1 stalk (white), predicted by the SMOG-ion model. SDF calculated from a simulation of the ribosome with an E-site tRNA present (orange). While there is clear accumulation of $\mathrm{Mg}^{2+}$ ions around the tRNA and L1 stalk, there is only a low density of $\mathrm{K}^{+}$ions (Fig. S9). b) Zoomed-in perspective of panel a. Visual inspection suggests $\mathrm{Mg}^{2+}$ ions can facilitate L1-tRNA association (proteins not shown). c) Average potential energy $\langle U\rangle$ of electrostatic interactions as a function of L1-tRNA separation $\left(R_{\text {L1-tRNA }}\right)$. Electrostatic energy is separately calculated for L1-tRNA interactions (blue), L1-ion interactions (orange), and tRNA-ion interactions (green). While direct L1tRNA electrostatics are repulsive, ion-mediated interactions are stronger and attractive. d) Probability distribution of the number of ions that are simultaneously within $10 \AA$ of the L1 stalk and E-site tRNA. As suggested by the SDF, there is a significantly larger number of $\mathrm{Mg}^{2+}$ ions, on average, at the L1-tRNA interface. This implicates diffuse $\mathrm{Mg}^{2+}$ ions are responsible for facilitating L1-tRNA association. 
$\mathrm{Mg}^{2+}$-mediate interactions.

\section{Discussion}

\section{Strategies for studying diffuse ions}

The accuracy of ionic models is often evaluated based on studies of the adenine riboswitch and 58-mer rRNA. The reason for this choice is that high-quality experimental measures are available that describe the distribution of monovalent $\left(\mathrm{K}^{+}\right.$and $\left.\mathrm{Cl}^{-}\right)$and divalent $\left(\mathrm{Mg}^{2+}\right)$ ions around these RNA. ${ }^{63,71}$ As described in the results, even though parameterization was based on experimental comparisons for a single ionic concentration, we find that the SMOGion model is able to capture the experimentally-measured concentration dependence of $\Gamma_{2+}$ for both systems. In terms of these common benchmarks, the SMOG-ion model provides a level of agreement that is comparable to other available models. Lammert et al. found that explicit-solvent simulations of the $58-$ mer $^{70}$ overestimated $\Gamma_{2+}$ by $2-3$. However, due to the small system size in that study, there was a significant uncertainty in the calculated bulk ion concentration. In terms of coarse-grained approaches, Nguyen et al. ${ }^{37}$ used a theory based on the reference interaction site model to develop effective potentials for divalent ions. This representation was able to predicted $\Gamma_{2+}$ to within a value of 1 for both systems, over the same ion concentration studied here. While their use of pair-wise effective potentials is similar to our approach, that model employed an implicit representation of monovalent ions. $\Gamma_{2+}$ values have also been accurately predicted using a generalized Manning condensation model proposed by Hayes et al. ${ }^{87}$ Overall, we find that the SMOG-ion model is able to predict $\Gamma_{2+}$ with accuracy that is comparable to this range of approaches.

While numerous models can accurately predict experimental $\Gamma_{2+}$ values, each is suited to address specific physical questions. In Nguyen et al., ${ }^{37}$ the objective of the study was to predict ion-dependent folding dynamics of RNA. Since folding can be described well with coarse-grained representations, a three-site model was appropriate in that case. Further, the 
model aimed to elucidate the dynamics of chelated ions during folding, which necessitated the proper treatment of inner-shell interactions with $\mathrm{Mg}^{2+}$. In the study of Lammert et al., ${ }^{70}$ explicit-solvent simulations were used to identify how small-scale (short time) structural fluctuations were correlated with individual ion association events. With this finely-detailed question in mind, a higher-resolution model was warranted. In contrast, Hayes et al. ${ }^{87}$ focused on more general questions pertaining to the utility of Manning theory to quantitatively predict ion condensation in asymmetric molecular systems, where an intermediate-resolution model is most suitable.

Here, our long-term objective is to establish a technique for studying large-scale structural dynamics that involve the transient formation of ion-mediated interactions. As an initial demonstration of this utility, we have described fluctuations about a single conformation of the ribosome. However, future work will seek to reveal how transient ion-association events can be correlated with large-scale (50-100 A) rearrangements. As discussed below, numerous studies have shown that detailed steric interactions strongly influence such motions in the ribosome. Accordingly, when constructing the SMOG-ion model, it was necessary to use an all-atom representation of the biomolecules, while coarse graining over the solvent.

\section{Modeling the factors that control biomolecular assemblies}

An outstanding challenge in the study of ribonucleoprotein assemblies is to precisely describe the influence of ions during large-scale conformational transitions. While there are efforts to refine ion parameters for use with explicit-solvent models, the large size of RNP assemblies (often MDa scales), combined with the slow timescales ( $\mu$ s-ms-s), makes direct simulation of the dynamics intractable with conventional models. In an effort to bridge our understanding of large-scale dynamics and ionic effects, we propose the SMOG-ion model. This model is less computationally demanding than explicit-solvent simulations, though simulations of large assemblies will nonetheless remain challenging. Fortunately, due to the simple functional form, the simulations scale to thousands of compute cores for large assemblies (Fig. S10). With 
this level of performance, modest-sized compute clusters are sufficient to perform simulations that describe millisecond effective timescales for MDa-scale assemblies. Accordingly, it is now tractable to explore the impact of ions on ensemble measures of the dynamics (e.g. average structures, RMSF, etc), as well as during large-scale conformational rearrangements. By building upon previous theoretical and computational advances, the current effort can enable the study of ionic effects in arbitrarily large and complex biomolecular assemblies.

For the SMOG-ion model, all-atom resolution was employed since previous studies have repeatedly shown how sterically-induced free-energy barriers can limit the kinetics of the ribosome. For example, multi-basin structure-based models have demonstrated how the steric composition of the A-site finger controls the scale of the free-energy barrier associated with $\mathrm{A} / \mathrm{P}$ hybrid formation. ${ }^{88}$ During $\mathrm{P} / \mathrm{E}$ hybrid formation, similar models have shown that the kinetics depends critically on the precise steric representation of the N-terminal tail of protein L33. ${ }^{89}$ In these examples, direct perturbations to the steric representation in the model revealed the central influence of excluded volume. Since the contribution of sterics will be robust, so long as atomic resolution is included, it is expected that the positions of these barriers will also be robust to the energetic details of the model. However, by applying more energetically-complete models, such as the SMOG-ion model, future studies will be able to further delineate the factors that control the scale of each barrier, and therefore the biological kinetics.

To complement the insights provided by the SMOG-ion model, explicit-solvent simulations and quantum mechanical methods can provide highly-detailed descriptions of biomolecular interactions within larger assemblies. For the ribosome, explicit-solvent models have been widely used to quantify energetics of bimolecular binding events, ${ }^{90}$ or small-scale (e.g. single-residue) structural rearrangements. ${ }^{91}$ In other studies, they have been used to quantify the stabilizing interactions between different components of the ribosome, which have included studies of the L1 stalk, ${ }^{84,86}$ interactions between the 3'-CCA tail of tRNA and its binding sites,${ }^{92}$ as well as between tRNA and the ribosome, ${ }^{93}$ synthetases ${ }^{94}$ and elongation 
factors. ${ }^{95}$ At an even higher level of resolution, quantum mechanical techniques are available to study chemical reactions. ${ }^{96,97}$

The stark differences between simplified/coarse-grained models and explicit-solvent/quantum techniques often makes direct comparison of the results unclear. Here, we present a model that is substantially more energetically detailed than traditional structure-based models, while also being far simpler than explicit-solvent models. We anticipate that these intermediateresolution models will help establish a more comprehensive understanding of RNP dynamics that will bridge the gap between detailed insights arising from explicit-solvent simulations and larger-scale dynamical processes that can be described by coarse-grained techniques.

\section{Concluding Remark}

Attributing a specific role to diffuse ions in ribonucleoprotein assemblies has remained elusive. While there has been notable progress in the study of monomeric systems, unambiguously identifying specific physical-chemical effects of diffuse ions in assemblies continues to be challenging. As a step in this direction, we developed and employed a model that provides an explicit treatment of non-Hydrogen atoms and ions, while providing an implicit treatment of solvent. We find that our model is able to capture experimental measures of the ionic environment for prototypical RNA systems, which motivated our application to a more complex system: the ribosome. By comparing the dynamics of the ribosome with a range of theoretical models, we identify specific conformational motions that are facilitated by diffuse-ion-mediated interactions. With this foundation, the study provides a theoretical and technical framework for identifying the ways in which diffuse ions help regulate the dynamics of complex biomolecular assemblies. 


\section{Methods}

\section{Structure-based "SMOG" model with explicit ions}

The SMOG-ion model is an all-atom structure-based "SMOG" model ${ }^{61,62}$ with explicit electrostatics and an explicit representation of diffuse ions $\left(\mathrm{K}^{+}, \mathrm{Cl}^{-}\right.$and $\left.\mathrm{Mg}^{2+}\right)$. Ionic interactions are defined in terms of Coulomb electrostatics and effective potentials. The potential energy may be described in terms of two components:

$$
V=V_{\mathrm{SMOG}}+V_{\mathrm{E}}
$$

$V_{\mathrm{SMOG}}$ refers to the all-atom structure-based potential energy and $V_{\mathrm{E}}$ describes the potential energy of all electrostatic interactions.

In the all-atom structure-based SMOG model $\left(V_{\mathrm{SMOG}}\right),{ }^{61,62}$ all non-hydrogen atoms are explicitly represented and an experimentally-identified configuration is defined as the global potential energy minimum. The functional form of the SMOG model is given by:

$$
\begin{aligned}
V_{\mathrm{SMOG}}= & \sum_{i j \in \text { bonds }} \frac{\epsilon_{\mathrm{b}}}{2}\left(r_{i j}-r_{i j}^{0}\right)^{2}+\sum_{i j k \in \text { angles }} \frac{\epsilon_{\theta}}{2}\left(\theta_{i j k}-\theta_{i j k}^{0}\right)^{2} \\
& +\sum_{i \in \text { improper }} \frac{\epsilon_{\chi \mathrm{imp}}}{2}\left(\chi_{i}-\chi_{i}^{0}\right)^{2}+\sum_{i \in \text { planar }} \frac{\epsilon_{\chi \mathrm{planar}}}{2}\left(\chi_{i}-\chi_{i}^{0}\right)^{2} \\
& +\sum_{i \in \text { backbone }} \epsilon_{\mathrm{BB}} F_{D}\left(\phi_{i}\right)+\sum_{i \in \text { sidechains }} \epsilon_{\mathrm{SC}} F_{D}\left(\phi_{i}\right) \\
& +\sum_{i j \in \text { contacts }} \epsilon_{\mathrm{C}}\left[\left(\frac{r_{i j}^{0}}{r_{i j}}\right)^{12}-2\left(\frac{r_{i j}^{0}}{r_{i j}}\right)^{6}\right] \\
& +\sum_{i j \notin \text { contacts }}\left(\frac{C_{18}}{r_{i j}^{18}}-\frac{C_{12}}{r_{i j}^{12}}\right)
\end{aligned}
$$

where

$$
F_{\mathrm{D}}\left(\phi_{i}\right)=\left[1-\cos \left(\phi_{\mathrm{i}}-\phi_{\mathrm{i}}^{0}\right)\right]+\frac{1}{2}\left[1-\cos \left(3\left(\phi_{\mathrm{i}}-\phi_{\mathrm{i}}^{0}\right)\right)\right]
$$

$\chi_{i}^{0}$ and $\phi_{i}^{0}$ are given the values found in a pre-assigned configuration. $r_{i j}^{0}$ and $\theta_{i j k}^{0}$ are assigned 
the corresponding values found in the Amber99sb-ildn force field, ${ }^{64}$ as employed in a previous SMOG-AMBER model. ${ }^{98}$ Interaction weights are assigned default values (consistent with previous studies (see ref 62). Contacts are defined using the Shadow Contact Map algorithm ${ }^{99}$ with a $6 \AA$ cutoff and a $1 \AA$ shadowing radius.

In contrast with previous SMOG models, we included a 12-18 potential for atom pairs that are not in contact in the experimentally-defined structure. This was introduced in order to define an excluded volume potential that mimics that of the AMBER force field, without including a deep attractive well (Fig. S11). With this approach, the steric representation provided by the model is consistent with the more highly-detailed AMBER model, while introducing a minimal degree of non-specific energetic roughness. The coefficients $C_{18}$ and $C_{12}$ were defined for each type of interaction based on fits to the corresponding 6-12 potentials in the Amber99sb-ildn force field ${ }^{64}$ (Fig. S11).

The electrostatic representation $\left(V_{\mathrm{E}}\right)$ includes direct Coulomb interactions $\left(V_{\text {coulomb}}\right)$, effective excluded volume potentials for diffuse ions $\left(V_{\text {ion-excl }}\right)$ and effective potentials that describe ionic solvation effects $\left(V_{\mathrm{sol}}\right)$ :

$$
\begin{aligned}
V_{\mathrm{E}} & =V_{\text {coulomb }}+V_{\text {ion-excl }}+V_{\text {sol }} \\
& =\sum_{i j} \frac{q_{i} q_{j}}{4 \pi \varepsilon \varepsilon_{0} r_{i j}}+\sum_{i j} \frac{A}{r_{i j}^{12}}+\sum_{i j}\left(\sum_{k=1}^{5} B^{(k)} e^{-C^{(k)}\left[r_{i j}-R^{(k)}\right]^{2}}\right) .
\end{aligned}
$$

$V_{\text {coulomb }}$ represents the direct Coulomb interactions between a pair of charges $q_{i}$ and $q_{j}$ with interatomic distance $r_{i j}$. $\varepsilon$ is the dielectric constant for water (80) and $\varepsilon_{0}$ is the permittivity of free space. The partial charge of each atom was derived from the Amber99sb-ildn forcefield. ${ }^{64}$ Since the SMOG-ion model only includes non-hydrogen atoms, the partial charge of each hydrogen atom in the AMBER force field was added to the corresponding non- $\mathrm{H}$ atom.

The effective excluded volume of each diffuse (hydrated) ion is account for by $V_{\text {ion-excl }}$. Consistent with previous efforts to model explicit ions in a coarse-grained model, ${ }^{50}$ pairwise potentials of the form $\frac{A}{r_{i j}^{12}}$ were used to define the excluded volume between ion $i$ with atom 
j. The parameter $A$ is different for each type of interaction in the model (e.g. Cl-Cl, Cl-K, K-O, etc). To simplify notation, subscripts are not shown. Following the protocol of Savelyev and Papoian, ${ }^{50}$ the values of the parameters $\{A\}$ were obtained through refinement based on comparison with explicit-solvent simulations (See Supporting Information for details).

The term $V_{\text {sol }}$ describes solvent-mediated ionic interactions, which manifest in the form of ionic shells. The functional form is the same as used previously, ${ }^{50}$ where a sum of Gaussians describe the shells (negative $B^{(k)}$ values) and intervening barriers (positive $B^{(k)}$ values). For each type of interaction considered, up to five Gaussians were included to describe up to three (outer) ionic shells. The location $\left(R^{(k)}\right)$ and width $\left(C^{(k)}\right)$ of each Gaussian was determined based on an initial fit to the corresponding radial distribution function. The amplitude of each Gaussian $\left(B^{(k)}\right)$ was refined based on comparison with explicit-solvent simulations and experimental measurements (details in Supporting Information). Consistent with the assignment of excluded volume parameters, unique Gaussian parameters were assigned to each type of modeled interaction.

Upon publication, the SMOG-ion model will be freely available through the SMOG 2 Force Field Repository (https://smog-server.org/smog2 - Force Field ID: AA_ions_Wang21.v1).

\section{Calculating preferential interaction coefficients}

We calculated the preferential interaction coefficient of $\mathrm{Mg}^{2+}$ ions $\left(\Gamma_{2+}\right)$ from simulations with the SMOG-ion model and used it as a metric to compare with experimental measurements. $\Gamma_{2+}$ describes the "excess" number of $\mathrm{Mg}^{2+}$ ions that accumulate around an RNA molecule due to electrostatic interactions. ${ }^{63,71,100-102}$ In the current study, $\Gamma_{2+}$ was calculated by taking the difference between the total number of simulated ions $\left(N_{\mathrm{Mg}^{2+}}\right)$ and the expected bulk value. The expected bulk number is the product of the bulk density $\left(\rho_{\mathrm{Mg}^{2+}}\right)$ and the volume of the simulated box $\left(V_{\mathrm{box}}\right)^{\mathrm{v}}$, such that $\Gamma_{2+}=N_{\mathrm{Mg}^{2+}}-\rho_{\mathrm{Mg}^{2+}} \times V_{\mathrm{box}}$. To calculate the bulk density, the RNA fragment was first recentered in the box for each frame of the

${ }^{\mathrm{v}}$ With the large dimensions of the simulated systems, the free volume (i.e. volume excluding the RNA) only differs from the total volume by less than $0.01 \%$. 
trajectory. The box was then partitioned into five equal-width $(\sim 140 \AA)$ slabs. The average density of $\mathrm{Mg}^{2+}$ was then calculated by excluding the central (RNA-containing) slab. For each system and ion concentration, the effective concentration was calculated for 20 independent replicas. The uncertainty in the concentration was defined as the standard deviation of the 20 replicate values. While simulating many replicas helped reduce the uncertainty (standard deviation of $\left[\mathrm{MgCl}_{2}\right] \sim 0.005 \mathrm{mM}$ ), due to the large dimensions of the simulated systems, this leads to a residual uncertainty of $\sim 1$ in $\rho_{\mathrm{Mg}^{2+}} \times V_{b o x}$. This uncertainty is then propagated to $\Gamma_{2+}$.

When calculating $\Gamma_{2+}$, it was necessary to account for chelated ions, as well as the diffuse ionic environment. Since the SMOG-ion was only parameterized to describe diffuse ions, strongly-bound chelated (inner-shell) ions were assigned a priori. In the adenine riboswitch, there are five $\mathrm{Mg}^{2+}$ binding sites (Fig.S4b) identified in the crystal structure (PDB: 1Y26). ${ }^{103}$ However, the binding site of Mg3 (Fig.S4b) is formed by crystallographic interactions ${ }^{103}$ and no divalent cation binding could be detected in the vicinity of this position through highresolution NMR spectroscopy and titration methods. ${ }^{104}$ Accordingly, this chelated ion was not included in the current simulations. However, since the other four $\mathrm{Mg}^{2+}$ ions are found deep within the grooves of the RNA, they were defined to be harmonically restrained to their chelation pockets. To compare with experimental approaches, which report the number of diffuse and bound ions, the four chelated ions were included in our calculation of $\Gamma_{2+}$. Chelated ions in the 58-mer are described in the results section.

\section{Supporting Information Available}

The following files are available free of charge.

- SI.pdf: Supporting methods and figures. 


\section{Author Information}

\section{Corresponding authors}

*Email:p.whitford@northeastern.edu

*Email:mohanty@bc.edu

\section{Author contributions}

All authors contributed to the design of the study. AW developed the model. AW and ML performed simulations. AW performed all analysis. All authors wrote the manuscript. UM and PCW supervised the project.

\section{Notes}

The authors declare no competing financial interest.

\section{Acknowledgement}

PCW was supported by NSF grant MCB-1915843. Work at the Center for Theoretical Biological Physics was also supported by the NSF (Grant PHY-2019745). We acknowledge generous support provided Northeastern University Discovery Cluster and the C3DDB cluster. We thank Yang Wang for comments and proofreading the manuscript.

\section{References}

(1) Pyle, A. M. Metal ions in the structure and function of RNA. J. Biol. Inorg. Chem. 2002, 7, 679-690.

(2) Woodson, S. A. Metal ions and RNA folding: a highly charged topic with a dynamic future. Curr. Opin. Chem. Biol. 2005, 9, 104-109.

(3) Draper, D. E. A guide to ions and RNA structure. RNA 2004, 10, 335-343. 
(4) Draper, D. E.; Grilley, D.; Soto, A. M. Ions and RNA folding. Annu. Rev. Biophys. Biomol. Struct. 2005, 34, 221-243.

(5) Schroeder, R.; Barta, A.; Semrad, K. Strategies for RNA folding and assembly. Nat. Rev. Mol. Cell Biol. 2004, 5, 908-919.

(6) Wan, Y.; Qu, K.; Ouyang, Z.; Kertesz, M.; Li, J.; Tibshirani, R.; Makino, D. L.; Nutter, R. C.; Segal, E.; Chang, H. Y. Genome-wide measurement of RNA folding energies. Mol. Cell 2012, 48, 169-181.

(7) Izatt, R. M.; Christensen, J. J.; Rytting, J. H. Sites and thermodynamic quantities associated with proton and metal ion interaction with ribonucleic acid, deoxyribonucleic acid, and their constituent bases, nucleosides, and nucleotides. Chem. Rev. 1971, $71,439-481$.

(8) Shamsi, M. H.; Kraatz, H. B. Interactions of metal ions with DNA and some applications. J. Inorg. Organomet. Polym. Mater. 2013, 23, 4-23.

(9) Abeysirigunawardena, S. C.; Woodson, S. A. Differential effects of ribosomal proteins and $\mathrm{Mg}^{2+}$ ions on a conformational switch during 30S ribosome 5'-domain assembly. RNA 2015, 21, 1859-1865.

(10) Kim, H. D.; Nienhaus, G. U.; Ha, T.; Orr, J. W.; Williamson, J. R.; Chu, S. Mg${ }^{2+}{ }_{-}$ dependent conformational change of RNA studied by fluorescence correlation and FRET on immobilized single molecules. Proc. Natl. Acad. Sci. USA 2002, 99, 42844289.

(11) Klein, D. J.; Moore, P. B.; Steitz, T. A. The contribution of metal ions to the structural stability of the large ribosomal subunit. RNA 2004, 10, 1366-1379.

(12) Gesteland, R. F. Unfolding of Escherichia coli ribosomes by removal of magnesium. J. Mol. Biol. 1966, 18, 356-371. 
(13) Kim, H. D.; Puglisi, J. D.; Chu, S. Fluctuations of transfer RNAs between classical and hybrid states. Biophys. J. 2007, 93, 3575-3582.

(14) Holtkamp, W.; Cunha, C. E.; Peske, F.; Konevega, A. L.; Wintermeyer, W.; Rodnina, M. V. GTP hydrolysis by EF-G synchronizes tRNA movement on small and large ribosomal subunits. EMBO J. 2014, 33, 1073-1085.

(15) Wohlgemuth, I.; Pohl, C.; Rodnina, M. V. Optimization of speed and accuracy of decoding in translation. EMBO J. 2010, 29, 3701-3709.

(16) Johansson, M.; Zhang, J.; Ehrenberg, M. Genetic code translation displays a linear trade-off between efficiency and accuracy of tRNA selection. Proc. Natl. Acad. Sci. USA 2012, 109, 131-136.

(17) Braunlin, W. H. NMR studies of cation-binding environments on nucleic acids. Adv. Biophys. Chem. 1995, 5, 89-140.

(18) Reid, S. S.; Cowan, J. A. Biostructural Chemistry of Magnesium Ion: Characterization of the Weak Binding Sites on tRNAPhe(yeast). Implications for Conformational Change and Activity. Biochemistry 1990, 29, 6025-6032.

(19) Cowan, J. A. Coordination chemistry of $\mathrm{Mg}^{2+}$ and 5S rRNA (Escherichia coli): Binding parameters, ligand symmetry, and implications for activity. J. Am. Chem. Soc. 1991, 113, 675-676.

(20) Kowerko, D.; König, S. L.; Skilandat, M.; Kruschel, D.; Hadzic, M. C.; Cardo, L.; Sigel, R. K. Cation-induced kinetic heterogeneity of the intron-exon recognition in single group II introns. Proc. Natl. Acad. Sci. U. S. A. 2015, 112, 3403-3408.

(21) Caminiti, R.; Licheri, G.; Piccaluga, G.; Pinna, G. X-ray diffraction study of $\mathrm{MgCl}_{2}$ aqueous solutions. J. Appl. Crystallogr. 1979, 12, 34-38. 
(22) Weingärtner, H.; Müller, K. J.; Hertz, H. G.; Edge, A. V.; Mills, R. Unusual behavior of transport coefficients in aqueous solutions of zinc chloride at $25^{\circ} \mathrm{C}$. J. Phys. Chem. 1984, 88, 2173-2178.

(23) Bleuzen, A.; Pittet, P. A.; Helm, L.; Merbach, A. E. Water exchange on magnesium(II) in aqueous solution: A variable temperature and pressure ${ }^{17} \mathrm{O}$ NMR study. Magn. Reson. Chem. 1997, 35, 765-773.

(24) Cole, P. E.; Yang, S. K.; Crothers, D. M. Conformational changes of transfer ribonucleic acid. Equilibrium phase diagrams. Biochemistry 1972, 11, 4358-4368.

(25) Urbanke, C.; Römer, R.; Maass, G. Tertiary structure of tRNA ${ }^{\text {Phe }}$ (Yeast): Kinetics and electrostatic repulsion. Eur. J. Biochem. 1975, 55, 439-444.

(26) Fuoss, R. M.; Katchalsky, A.; Lifson, S. The potential of an infinite rod-like molecule and the distribution of the counter ions. Proc. Natl. Acad. Sci. USA 1951, 37, 579-589.

(27) Honig, B.; Nicholls, A. Classical electrostatics in biology and chemistry. Science 1995, 268, 1144-1149.

(28) Forsman, J. A simple correlation-corrected Poisson-Boltzmann theory. J. Phys. Chem. $B$ 2004, 108, 9236-9245.

(29) Manning, G. S. The molecular theory of polyelectrolyte solutions with applications to the electrostatic properties of polynucleotides. Q. Rev. Biophys. 1978, 11, 179-246.

(30) Mohanty, U.; Ninham, B. W.; Oppenheim, I. Dressed polyions, counterion condensation, and adsorption excess in polyelectrolyte solutions. Proc. Natl. Acad. Sci. USA 1996, 93, 4342-4344.

(31) Manning, G. S.; Mohanty, U. Counterion condensation on ionic oligomers. Phys. A: Stat. Mech. and its Appl. 1997, 247, 196-204. 
(32) Mohanty, U.; Spasic, A.; Kim, H. D.; Chu, S. Ion atmosphere of three-way junction nucleic acid. J. Phys. Chem. B 2005, 109, 21369-21374.

(33) Taubes, C. H.; Mohanty, U.; Chu, S. Ion atmosphere around nucleic acid. J. Phys. Chem. B 2005, 109, 21267-21272.

(34) Spasic, A.; Mohanty, U. Counterion condensation in nucleic acid. Adv. Chem. Phys. 2008, 139, 139-176.

(35) Hirata, F.; Rossky, P. J. An extended rism equation for molecular polar fluids. Chem. Phys. Lett. 1981, 83, 329-334.

(36) Pettitt, B. M.; Rossky, P. J. Integral equation predictions of liquid state structure for waterlike intermolecular potentials. J. Chem. Phys. 1982, 7r, 1451-1457.

(37) Nguyen, H. T.; Hori, N.; Thirumalai, D. Theory and simulations for RNA folding in mixtures of monovalent and divalent cations. Proc. Natl. Acad. Sci. USA 2019, 116, $21022-21030$.

(38) Kjellander, R. Modified Debye-Hückel approximation with effective charges: An application of dressed ion theory for electrolyte solutions. J. Phys. Chem. 1995, 99, $10392-10407$.

(39) Frusawa, H.; Hayakawa, R. Generalizing the Debye-Hückel equation in terms of density functional integral. Phys. Rev. E 2000, 61, R6079-R6082.

(40) Denesyuk, N. A.; Thirumalai, D. Coarse-grained model for predicting RNA folding thermodynamics. J. Phys. Chem. B 2013, 117, 4901-4911.

(41) Schaefer, M.; Karplus, M. A comprehensive analytical treatment of continuum electrostatics. J. Phys. Chem. 1996, 100, 1578-1599.

(42) Bashford, D.; Case, D. A. Generalized born models of macromolecular solvation effects. Annu. Rev. Phys. Chem. 2000, 51, 129-152. 
(43) Murthy, C. S.; Bacquet, R. J.; Rossky, P. J. Ionic distributions near polyelectrolytes. A comparison of theoretical approaches. J. Phys. Chem. 1985, 89, 701-710.

(44) Netz, R. R.; Orland, H. Beyond Poisson-Boltzmann: Fluctuation effects and correlation functions. Eur. Phys. J. E 2000, 1, 203-214.

(45) Attard, P. Electrolytes and the electric double layer. Adv. Chem. Phys. 1996, 92, $1-159$.

(46) Shi, Y. Z.; Wang, F. H.; Wu, Y. Y.; Tan, Z. J. A coarse-grained model with implicit salt for RNAs: Predicting 3D structure, stability and salt effect. J. Chem. Phys. 2014, 141, $1051021-13$.

(47) Marcovitz, A.; Levy, Y. Frustration in protein-DNA binding influences conformational switching and target search kinetics. Proc. Natl. Acad. Sci. U. S. A. 2011, 108, 1795717962.

(48) Givaty, O.; Levy, Y. Protein sliding along DNA: Dynamics and structural characterization. J. Mol. Biol. 2009, 385, 1087-1097.

(49) Wong, G. C.; Pollack, L. Electrostatics of strongly charged biological polymers: Ionmediated interactions and self-organization in nucleic acids and proteins. Annu. Rev. Phys. Chem. 2010, 61, 171-189.

(50) Savelyev, A.; Papoian, G. A. Molecular renormalization group coarse-graining of electrolyte solutions: Application to aqueous $\mathrm{NaCl}$ and $\mathrm{KCl}$. J. Phys. Chem. B 2009, $113,7785-7793$.

(51) Savelyev, A.; Papoian, G. A. Chemically accurate coarse graining of double-stranded DNA. Proc. Natl. Acad. Sci. USA 2010, 10\%, 20340-20345.

(52) Denesyuk, N. A.; Thirumalai, D. How do metal ions direct ribozyme folding? Nat. Chem. 2015, 7, 793-801. 
(53) Åqvist, J. Ion-water interaction potentials derived from free energy perturbation simulations. J. Phys. Chem. 1990, 94, 8021-8024.

(54) Joung, I. S.; Cheatham, III, T. E. Determination of alkali and halide monovalent ion parameters for use in explicitly solvated biomolecular simulations. J. Phys. Chem. B 2008, 112, 9020-9041.

(55) Chen, A. A.; Pappu, R. V. Parameters of monovalent ions in the AMBER-99 forcefield:Assessment of inaccuracies and proposed improvements. J. Phys. Chem. B 2007, $111,11884-11887$.

(56) Kirmizialtin, S.; Pabit, S. A.; Meisburger, S. P.; Pollack, L.; Elber, R. RNA and its ionic cloud: Solution scattering experiments and atomically detailed simulations. Biophys. J. 2012, 102, 819-828.

(57) Chen, A. A.; García, A. E. High-resolution reversible folding of hyperstable RNA tetraloops using molecular dynamics simulations. Proc. Natl. Acad. Sci. USA 2013, 110, 16820-16825.

(58) Miner, J. C.; García, A. E. Concentration-dependent and configuration-dependent interactions of monovalent ions with an RNA tetraloop. J. Chem. Phys. 2018, 148, 222837 1-11.

(59) Srivastava, A.; Timsina, R.; Heo, S.; Dewage, S. W.; Kirmizialtin, S.; Qiu, X. Structure-guided DNA-DNA attraction mediated by divalent cations. Nucleic Acid Res. 2020, 48, 7018-7026.

(60) Arenz, S.; Bock, L. V.; Graf, M.; Innis, C. A.; Beckmann, R.; Grubmüller, H.; Vaiana, A. C.; Wilson, D. N. A combined cryo-EM and molecular dynamics approach reveals the mechanism of ErmBL-mediated translation arrest. Nat. Commun. 2016, 7, 1-14. 
(61) Whitford, P. C.; Noel, J. K.; Gosavi, S.; Schug, A.; Sanbonmatsu, K. Y.; Onuchic, J. N. An all-atom structure-based potential for proteins: Bridging minimal models with allatom empirical forcefields. Prot. Struct. Func. Bioinfo. 2009, 75, 430-441.

(62) Noel, J. K.; Levi, M.; Raghunathan, M.; Lammert, H.; Hayes, R. L.; Onuchic, J. N.; Whitford, P. C. SMOG 2: A versatile software package for generating structure-based models. PLoS Comput. Biol. 2016, 12, e1004794 1-14.

(63) Grilley, D.; Misra, V.; Caliskan, G.; Draper, D. E. Importance of partially unfolded conformations for $\mathrm{Mg}^{2+}$-induced folding of RNA tertiary structure: Structural models and free energies of $\mathrm{Mg}^{2+}$ interactions. Biochem. 2007, 46, 10266-10278.

(64) Lindorff-Larsen, K.; Piana, S.; Palmo, K.; Maragakis, P.; Klepeis, J. L.; Dror, R. O.; Shaw, D. E. Improved side-chain torsion potentials for the Amber ff99SB protein force field. Prot. Struct. Func. Bioinfo. 2010, 78, 1950-1958.

(65) Conn, G. L.; Gittis, A. G.; Lattman, E. E.; Misra, V. K.; Draper, D. E. A compact RNA tertiary structure contains a buried backbone- $\mathrm{K}^{+}$complex. J. Mol. Biol. 2002, 318, 963-973.

(66) Hayes, R. L.; Noel, J. K.; Mohanty, U.; Whitford, P. C.; Hennelly, S. P.; Onuchic, J. N.; Sanbonmatsu, K. Y. Magnesium fluctuations modulate RNA dynamics in the SAM-I riboswitch. J. Am. Chem. Soc. 2012, 134, 12043-12053.

(67) Hayes, R. L.; Noel, J. K.; Whitford, P. C.; Mohanty, U.; Sanbonmatsu, K. Y.; Onuchic, J. N. Reduced model captures $\mathrm{Mg}^{2+}-\mathrm{RNA}$ interaction free energy of riboswitches. Biophys. J. 2014, 106, 1508-1519.

(68) Misra, V. K.; Draper, D. E. A thermodynamic framework for $\mathrm{Mg}^{2+}$ binding to RNA. Proc. Natl. Acad. Sci. USA 2001, 98, 12456-12461. 
(69) Xi, K.; Wang, F. H.; Xiong, G.; Zhang, Z. L.; Tan, Z. J. Competitive binding of $\mathrm{Mg}^{2+}$ and $\mathrm{Na}^{+}$ions to nucleic acids: from helices to tertiary structures. Biophys. J. 2018, $114,1776-1790$.

(70) Lammert, H.; Wang, A.; Mohanty, U.; Onuchic, J. N. RNA as a complex polymer with coupled dynamics of ions and water in the outer solvation sphere. J. Phys. Chem. B 2018, 122, 11218-11227.

(71) Leipply, D.; Draper, D. E. Effects of $\mathrm{Mg}^{2+}$ on the free energy landscape for folding a purine riboswitch RNA. Biochem. 2011, 50, 2790-2799.

(72) Rozov, A.; Khusainov, I.; El Omari, K.; Duman, R.; Mykhaylyk, V.; Yusupov, M.; Westhof, E.; Wagner, A.; Yusupova, G. Importance of potassium ions for ribosome structure and function revealed by long-wavelength X-ray diffraction. Nat. Commun. 2019, 10, 1-12.

(73) Jackson, J.; Nguyen, K.; Whitford, P. C. Exploring the balance between folding and functional dynamics in proteins and RNA. Int. J. Mol. Sci. 2015, 16, 6868-6889.

(74) Rodnina, M. V.; Wintermeyer, W. Fidelity of aminoacyl-tRNA selection on the ribosome: kinetic and structural mechanisms. Annu. Rev. Biochem. 2001, 70, 415-435.

(75) Johansson, M.; Lovmar, M.; Ehrenberg, M. Rate and accuracy of bacterial protein synthesis revisited. Curr. Opin. Microbio. 2008, 11, 141-147.

(76) Valle, M.; Zavialov, A.; Sengupta, J.; Rawat, U.; Ehrenberg, M.; Frank, J. Locking and unlocking of ribosomal motions. Cell 2003, 114, 123-134.

(77) Frank, J.; Gonzalez, R. L., Jr. Structure and dynamics of a processive Brownian motor: The translating ribosome. Annu. Rev. Biochem. 2010, 79, 381-412.

(78) Agirrezabala, X.; Liao, H. Y.; Schreiner, E.; Fu, J.; Ortiz-Meoz, R. F.; Schulten, K.; 
Green, R.; Frank, J. Structural characterization of mRNA-tRNA translocation intermediates. Proceedings of the National Academy of Sciences 2012, 109, 6094-6099.

(79) Fei, J.; Bronson, J. E.; Hofman, J. M.; Srinivas, R. L.; Wiggins, C. H.; Gonzalez, R. L. Allosteric collaboration between elongation factor $\mathrm{G}$ and the ribosomal L1 stalk directs tRNA movements during translation. Proc. Natl. Acad. Sci. USA 2009, 106, 1570215707.

(80) Fei, J.; Kosuri, P.; MacDougall, D. D.; Gonzalez, R. L. Coupling of ribosomal L1 stalk and tRNA dynamics during translation elongation. Mol. Cell 2008, 30, 348-359.

(81) Cornish, P. V.; Ermolenko, D. N.; Staple, D. W.; Hoang, L.; Hickerson, R. P.; Noller, H. F.; Ha, T. Following movement of the L1 stalk between three functional states in single ribosomes. Proc. Natl. Acad. Sci. USA 2009, 106, 2571-2576.

(82) Tama, F.; Valle, M.; Frankt, J.; Brooks, C. L. Dynamic reorganization of the functionally active ribosome explored by normal mode analysis and cryo-electron microscopy. Proc. Natl. Acad. Sci. USA 2003, 100, 9319-9323.

(83) Wang, Y.; Rader, A. J.; Bahar, I.; Jernigan, R. L. Global ribosome motions revealed with elastic network model. J. Struct. Biol. 2004, 147, 302-314.

(84) Trabuco, L. G.; Schreiner, E.; Eargle, J.; Cornish, P.; Ha, T.; Luthey-Schulten, Z.; Schulten, K. The role of L1 stalk-tRNA interaction in the ribosome elongation cycle. J. Mol. Biol. 2010, 402, 741-760.

(85) Mohan, S.; Noller, H. F. Recurring RNA structural motifs underlie the mechanics of L1 stalk movement. Nat. Commun. 2017, 8, 1-11.

(86) Bock, L. V.; Blau, C.; Schröder, G. F.; Davydov, I. I.; Fischer, N.; Stark, H.; Rodnina, M. V.; Vaiana, A. C.; Grubmüller, H. Energy barriers and driving forces in tRNA translocation through the ribosome. Nat. Struct. Mol. Biol. 2013, 20, 1390-1396. 
(87) Hayes, R. L.; Noel, J. K.; Mandic, A.; Whitford, P. C.; Sanbonmatsu, K. Y.; Mohanty, U.; Onuchic, J. N. Generalized Manning condensation model captures the RNA ion atmosphere. Phys. Rev. Lett. 2015, 114, 258105 1-6.

(88) Nguyen, K.; Yang, H.; Whitford, P. C. How the ribosomal A-site finger can lead to tRNA species-dependent dynamics. J. Phys. Chem. B 2017, 121, 2767-2775.

(89) Levi, M.; Walak, K.; Wang, A.; Mohanty, U.; Whitford, P. C. A steric gate controls hybrid-state formation of tRNA on the ribosome. Nat. Commun. 2020, 11, 1-12.

(90) Trobro, S.; Aqvist, J. Role of ribosomal protein L27 in peptidyl transfer. Biochemistry 2008, 47, 4898-4906.

(91) Vaiana, A. C.; Sanbonmatsu, K. Y. Stochastic gating and drug-ribosome interactions. J Mol Biol 2009, 386, 648-661.

(92) Johansson, M.; Ieong, K. W.; Trobro, S.; Strazewski, P.; Åqvist, J.; Pavlov, M. Y.; Ehrenberg, M. pH-sensitivity of the ribosomal peptidyl transfer reaction dependent on the identity of the A-site aminoacyl-tRNA. Proc. Natl. Acad. Sci. USA 2011, 108, $79-84$.

(93) Bock, L. V.; Blau, C.; Vaiana, A. C.; Grubmüller, H. Dynamic contact network between ribosomal subunits enables rapid large-scale rotation during spontaneous translocation. Nucleic Acid Res. 2015, 43, 6747-6760.

(94) Sethi, A.; Eargle, J.; Black, A. A.; Luthey-Schulten, Z. Dynamical networks in tRNA:protein complexes. Proc. Natl. Acad. Sci. USA 2009, 106, 6620-6625.

(95) Eargle, J.; Black, A. A.; Sethi, A.; Trabuco, L. G.; Luthey-Schulten, Z. Dynamics of Recognition between tRNA and elongation factor Tu. J Mol Biol 2008, 37r, 13821405. 
(96) Trobro, S.; Åquist, J. Mechanism of peptide bond synthesis on the ribosome. Proc. Natl. Acad. Sci. USA 2005, 102, 12396-12400.

(97) Gindulyte, A.; Bashan, A.; Agmon, I.; Massa, L.; Yonath, A.; Karle, J. The transition state for formation of the peptide bond in the ribosome. Proc. Natl. Acad. Sci. USA 2007, 103, 13327-13332.

(98) Whitford, P. C.; Jiang, W.; Serwer, P. Simulations of phage T7 capsid expansion reveal the role of molecular sterics on dynamics. Viruses 2020, 12, 1273 1-14.

(99) Noel, J. K.; Whitford, P. C.; Onuchic, J. N. The shadow map: A general contact definition for capturing the dynamics of biomolecular folding and function. J. Phys. Chem. B 2012, 116, 8692-8702.

(100) Timasheff, S. N. Water as ligand: Preferential binding and exclusion of denaturants in protein unfolding. Biochem. 1992, 31, 9857-9864.

(101) Eisenberg, H. Biological macromolecules and polyelectrolytes in solution; Clarendon Press, 1976.

(102) Rice, S. A.; Nagasawa, M. Polyelectrolyte Solutions; Academic Press: New York, 1961.

(103) Serganov, A.; Yuan, Y.-R.; Pikovskaya, O.; Polonskaia, A.; Malinina, L.; Phan, A. T.; Hobartner, C.; Micura, R.; Breaker, R. R.; Patel, D. J. Structural basis for discriminative regulation of gene expression by adenine- and guanine-sensing mRNAs. Chem. Biol. 2004, 11, 1729-1741.

(104) Noeske, J.; Schwalbe, H.; Wöhnert, J. Metal-ion binding and metal-ion induced folding of the adenine-sensing riboswitch aptamer domain. Nuc. Acids Res. 2007, 35, 52625273. 